Revista Ibero-Americana de Ciências Ambientais

Ibero-American Journal of Environmental Science

Anais do Simpósio Interdisciplinar de Práticas de Produção

Agrícola e Conservação Ambiental - Jan 2017 - v.8 - n.1

\title{
Pegada hídrica como medida sustentável na produção de Metarhizium Anisopliae (Metsch.) Sorok para a canavicultura
}

Em tempos de busca pela sustentabilidade hídrica faz-se essencial o desenvolvimento de técnicas e aplicações de métodos de reuso de água em sistemas produtivos. O consumo de água em áreas de suporte da cadeia produtiva da cana-de-açúcar é fator fundamental no desperdício hídrico, cujos estudos de potencialização da pegada hídrica são incipientes. Objetivou-se aplicar um sistema de reuso de água no processo de produção do fungo Metarhizium anisopliae, visando à otimização da pegada hídrica no controle biológico da cigarrinha no cultivo da cana-deaçúcar. $O$ estudo deu-se em laboratório de produção de fungos de uma usina sucroenergética situada no município de Nova Olímpia, estado de Mato Grosso. Quantificou-se o consumo de água e seu desperdício relacionado a cada atividade, através de testes expeditos. A implantação do reuso de água no sistema de destilação, principal agente do desperdício, reduziu o consumo em $51,21 \%$. 0 processo produtivo colaborou com cerca de $97 \%$ da oferta de água de reuso mensal. Esse valor poderia suprir de maneira individual toda a necessidade mensal de água do laboratório (37.980 litros). Embora não opere em circuito fechado, se testes de potabilidade fossem adotados, o sistema poderia ser modificado para este fim, tornando-o sustentável. A adoção de medidas de reuso de água, portanto, apresentou-se como uma técnica eficiente à conservação da água e a consequente melhoria da pegada hídrica azul como forma de medida de sustentabilidade ambiental.

Palavras-chave: Cana-de-Açúcar; Pegada Hídrica Azul; Recursos Naturais; Reuso de Água; Sustentabilidade.

\section{Water footprint how sustainable measure in production of Metarhizium Anisopliae (Metsch.) Sorok for sugar cane culture}

\begin{abstract}
In times of search for water sustainability, it is essential to develop techniques and applications of water reuse methods in production systems. Water consumption in support areas of production of sugarcane chain is a key factor in water waste, whose potentiation studies of water footprint are incipient. The objective was to apply a water reuse system in Metarhizium anisopliae fungus production process aimed at optimizing the water footprint in the biological control of the cultivation of sugarcane. The study took place in fungal production laboratory of a sugarcane mill in the municipality of Nova Olimpia, State of Mato Grosso. It was quantitated water consumption and wastage associated with each activity through expeditious testing. The implementation of water reuse in the distillation system, main waste agent, reduced consumption by $51.21 \%$. The production process has collaborated with about $97 \%$ of the monthly recycled water supply. This value could take individually all the monthly need for laboratory water (37,980 liters). Although not operate in closed circuit, if potability tests were adopted, the system could be modified for this purpose, making it sustainable. The adoption of water reuse measures, therefore, presented itself as an effective technique to water conservation and the improvement of the blue water footprint as a form of environmental sustainability measure.
\end{abstract}

Keywords: Sugar Cane; Blue Water Footprint; Natural Resources; Water Reuse; Sustainability.

Topic: Química Agrícola e Ambiental

Reviewed anonymously in the process of blind peer.

Thiziane Helen Lorenzon

Universidade do Estado de Mato Grosso, Brasil

http://lattes.cnpq.br/1565762109716372

thiziane@hotmail.com

Luiz Carlos Machado Filho

Universidade Candido Mendes, Brasi

http://lattes.cnpq.br/6391634475153827

luiz.carlos@uisanet.com.br

Sandra Mara Alves da Silva Neves

Universidade Federal de Mato Grosso, Brasil

http://lattes.cnpq.br/6430066425008976

ssneves@unemat.br

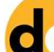

DOI: 10.6008/SPC2179-6858.2017.001.0008
Received: 20/04/2016

Approved: 25/05/2016

\author{
Rogério Pontes Xavier \\ Universidade Federal Rural do Rio de Janeiro, Brasi \\ http://lattes.cnpq.br/5045624481236222
}

xavierrogerio@hotmail.com

Referencing this:

LORENZON, T. H.; MACHADO FILHO, L. C.; NEVES, S. M. A. S.; XAVIER, R. P.. Pegada hídrica como medida sustentável na produção de Metarhizium Anisopliae (Metsch.) Sorok para a canavicultura. Revista Ibero-Americana de Ciências Ambientais, v.8, n.1, p.91-98, 2017. DOI: http://doi.org/10.6008/SPC2179-6858.2017.001.0008 


\section{INTRODUÇÃO}

A água, recurso natural finito, é fator limitante indispensável para a manutenção da vida em âmbito social, econômico e ambiental. A atual demanda desse recurso, amparada às estruturas de planejamento e administração dos Conselhos Nacionais e Estaduais de Recursos Hídricos, nos termos da lei n . 9.433/1997 (BRASIL, 1997), a qual estabelece a cobrança pelo uso da água, tem sido uma forma de atenuar as implicações à finitude e à qualidade desse bem.

A escassez da oferta hídrica vem impactando de maneira direta no desenvolvimento do processo produtivo agrícola. Segundo estudos de Bertoncini (2008), agroindústrias, como as usinas produtoras de açúcar e álcool, são grandes tomadoras de água. A grande crise da água, prevista para o ano de 2020 (WREGE, 2000) tem preocupado cientistas de diversas áreas do mundo e o caminho que poderá conduzir ao caos hídrico já é trilhado (MORAES e JORDÃO, 2002).

O consumo de água em áreas de suporte da cadeia produtiva da canavicultura é um aspecto determinante dentro do seu nicho de atividades, sendo importante variável da pegada hídrica, na produção do fungo Metarhizium anisopliae com fins de controle biológico para combate da cigarrinha (Mahanarva fimbriolata), praga da cultura de cana-de-açúcar.

A interdependência do processo produtivo com o uso de água vem favorecendo a aplicação da gestão ambiental focada na conservação hídrica. Segundo estudos da FIESP (2011), a gestão ambiental pode ser compreendida como as práticas, técnicas e tecnologias que aperfeiçoam a eficiência do uso da água.

A Agência Nacional das Águas (BRASIL, 2005) reporta a necessidade em se restabelecer o equilíbrio entre a oferta e demanda de água a fim de que se garanta a sustentabilidade do desenvolvimento econômico e social, dando-se por meio de métodos e sistemas alternativos específicos às características de sistemas e centros de produção.

A manutenção do equilíbrio entre as sustentabilidades socioeconômica e ambiental requer não apenas uma compreensão dos fluxos econômicos, mas também o conhecimento de quanto da capacidade biológica é necessário para absorver os impactos ambientais produzidos pela humanidade (SILVA et al., 2013).

A utilização da pegada hídrica como indicador de conservação ambiental, amplamente aplicado no mundo todo (ZHAO et al., 2009; ROMAGUERA et al., 2010; FENG et al., 2011) é, no entanto, incipiente nos processos produtivos agrícolas do Brasil (SILVA et al., 2013). Partindo desse pressuposto, a determinação da pegada hídrica é capaz de quantificar o consumo de água total ao longo da cadeia produtiva (YU et al., 2010). Nesse sentido, a crescente demanda por água tratada tem feito do reuso planejado de água um tema atual e de grande importância (MACHADO, 2004).

Tendo em vista que o controle biológico seja uma técnica firmada nos conceitos agroecológicos de combate às pragas agrícolas, é de suma importância que a cadeia produtiva dos agentes biológicos seja sustentável. Nesse sentido, faz-se essencial a minimização do consumo de água de forma que a pegada hídrica do setor do agronegócio seja eficiente. 
Para tanto, objetivou-se aplicar um sistema de reuso de água no processo de produção do fungo Metarhizium anisopliae, visando à otimização da pegada hídrica no controle biológico do cultivo da cana-deaçúcar na perspectiva de servir como uma medida de sustentabilidade ambiental.

\section{METODOLOGIA}

\section{Área de Estudo}

O estudo foi realizado em um laboratório de produção do fungo $M$. anisopliase de uma usina sucroenergética de grande porte localizada no município de Nova Olímpia/MT (Figura 1), integrante da região oeste de planejamento do estado de Mato Grosso (MATO GROSSO, 2012).

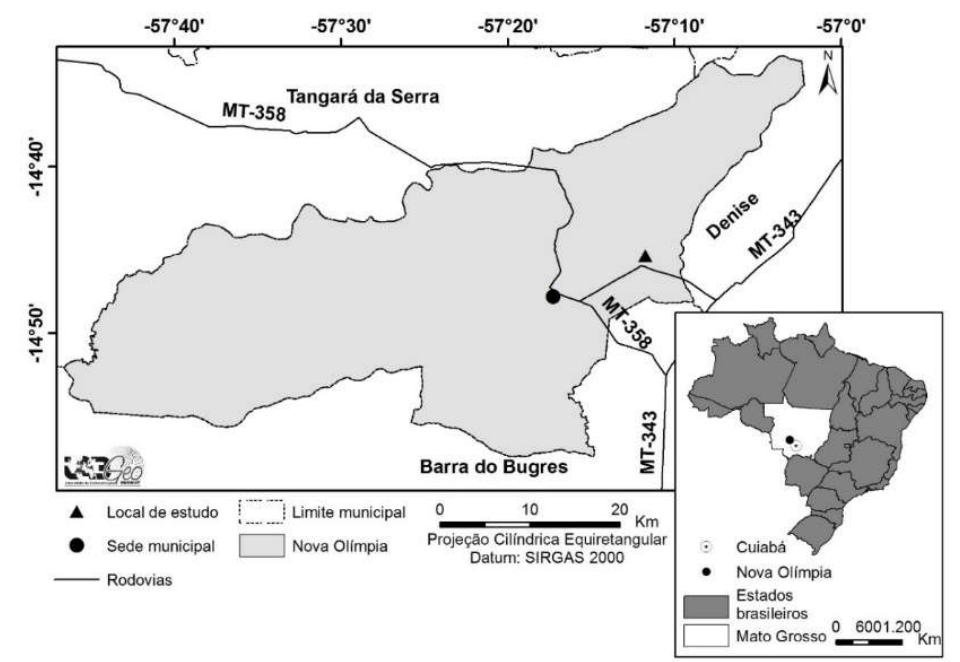

Figura 1: Área de estudo no contexto estadual e municipal. Fonte: LABGEO UNEMAT (2016).

A alimentação de água do laboratório é proveniente de captação no Rio Angelim, conforme outorga de direto de uso de recurso hídrico concedida pelo órgão ambiental do estado de Mato Grosso. A água passa por uma Estação de Tratamento de Água - ETA, recalcada para o suprimento da área industrial e direcionada a um reservatório de $7.000 \mathrm{~m}^{3}$ interligado a outro de $2.000 \mathrm{~m}^{3}$, o qual realiza a distribuição para os setores de produção de fungo.

A produção do fungo M. anisopliase chega à marca de $4.500 \mathrm{~kg}$ substrato/ano, considerando as áreas infestadas com a praga Mahanarva fimbriolata para um total de 70.000 ha de cultivo de cana-de-açúcar, inseridos na sub-bacia do rio Branco pertencente à Bacia do Alto Paraguai, onde encontra-se situado o Pantanal Mato-grossense.

\section{Procedimentos Metodológicos}

Para subsidiar o trabalho foi realizada uma visita técnica para a coleta de informações físicas e funcionais do uso da água e verificação da existência de desperdícios nas atividades laboratoriais através de testes expeditos, conforme metodologia de Silva (2004). 
Postos os dados, realizou-se a quantificação do consumo de água através da metodologia proposta por Oliveira (1999), com base no coeficiente de uso (TOMAZ, 2000), para a determinação da pegada hídrica utilizada no processo, seguindo a fórmula:

$$
P H=\frac{L}{A c} \times D
$$

Em que:

$P H=$ Pegada Hídrica

$L=$ Litros

$A c=$ Agente consumidor

$D=$ Dia

Estabelecidos os números, identificou-se o processo produtivo do fungo M. anisopliae como a maior fonte de desperdício de água. Dessa forma, aplicou-se um sistema de reuso de água no processo de destilação conforme a NBR 13969:1997 e NBR 5626:1998, para uma $Q_{\text {máx }} 150$ L. $^{-1}$ conduzida através de fluxo gravitacional por tubulação de PVC rígido $(60 \mathrm{~mm})$ a um reservatório de fibra apoiado em base de concreto, dupla ferragem, com capacidade de $6.000 \mathrm{~L}$. A água reutilizada no processo sai com $Q_{\text {máx }}=1,25 \mathrm{~L} . \mathrm{S}^{-}$ ${ }^{1}$ através de sucção de bomba centrífuga de $1 / 3 \mathrm{CV}$, conforme sugerido por Creder (2006) e representado na figura 2 .

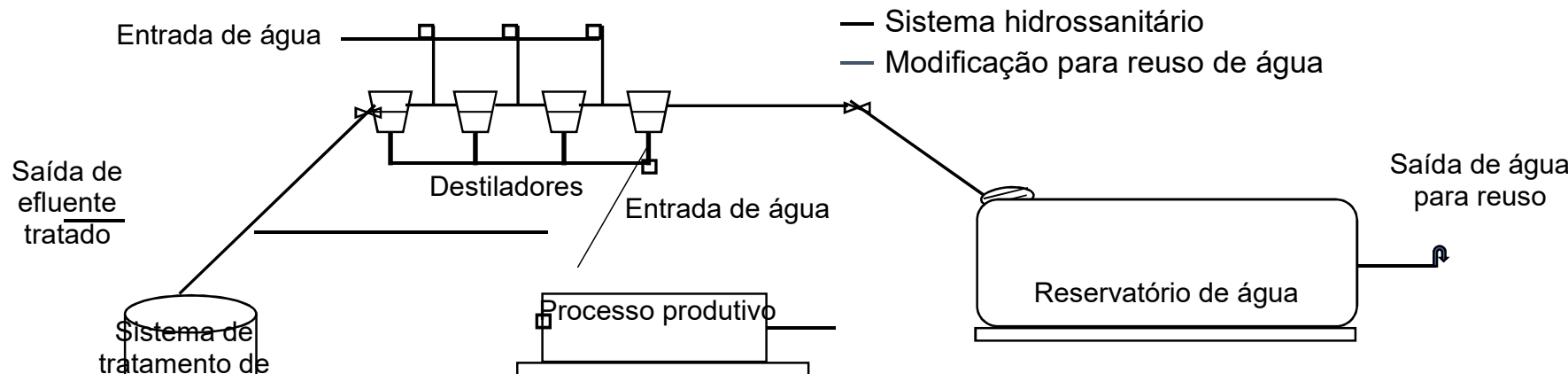

Figura 2: Sistema hidrossanitário do laboratório com modificação para reuso de água.

\section{RESULTADOS E DISCUSSÕES}

A avaliação técnica da estrutura física e funcional do uso de água mostrou que as atividades laboratoriais consomem 162.360 L/mês ${ }^{-1}$ e apresentam um desperdício de 76,60\% (Tabela 1).

Tabela 1: Uso de água por atividade no laboratório de fungos.

\begin{tabular}{lccc}
\hline \multicolumn{1}{c}{ Atividades } & $\begin{array}{c}\text { Pegada hídrica } \\
\left(\text { L.dia }^{-1}\right)\end{array}$ & $\begin{array}{c}\text { Pegada hídrica } \\
\left(\text { L.mês }^{-1}\right)\end{array}$ & $\begin{array}{c}\text { Desperdício } \\
(\text { L.mês }\end{array}$ \\
\hline Processo produtivo & 5.190 & 124.560 & 111.360 \\
Atividade de limpeza & 2.250 & 54.000 & 9.000 \\
Consumo humano & 1.200 & 28.800 & 11.520 \\
\hline Total & 8.640 & 207.360 & 131.880 \\
\hline
\end{tabular}

Esse resultado é atribuído à falta de controle e planejamento do uso racional desse recurso, essencial para a produção de fungo. Segundo os preceitos de Mierzwa e Hespanhol (2005) é importante a criação de estratégias que compatibilizem o uso da água nas atividades humanas à ideia de que os recursos hídricos não 
são abundantes. Tomaz (2001) salienta que a conservação da água é um conjunto de atividades com o objetivo de reduzir a demanda de água, melhorar o uso da água e reduzir as perdas e desperdícios da mesma.

No processo produtivo, mais de $89 \%$ do consumo mensal é desperdiçado. Para a produção diária de $225 \mathrm{~kg}$ de arroz, principal substrato para a proliferação do fungo, faz-se uso de 160 litros de água destilada e 390 litros de água autoclavada.

O processo de destilação no laboratório teve sua pegada hídrica caracterizada como negativa, considerando que se produz $30 \mathrm{~L}$ de água residual para $1 \mathrm{~L}$ de água destilada. Em estudo de Marsaro e Guimarães (2007), verificou-se que para produzir 1 litro de água destilada, em média, 17 a 21 litros de água potável são desperdiçados. Essas variações, no entanto, podem ser atribuídas à marca e ao modelo do equipamento utilizado na destilação. Nunes (2006), reforçaram que estes equipamentos necessitam de um grande volume de água para o processo de destilação, sendo que uma pequena parte se transforma em água destilada e o restante é utilizado para resfriamento.

A atividade de limpeza é responsável por cerca de $26 \%$ do consumo diário de água, caracterizandoo como o segundo posicionado. Em contrapartida, em se tratando do consumo mensal, esta é a atividade com menor participação no percentual total. Perdas de água implicam na necessidade de uma maior produção para atender à mesma demanda (TSUTIYA, 2005).

Infere-se que $16,66 \%$ do consumo mensal são desperdiçados, demonstrando uma pegada hídrica insustentável, o que reflete uma preocupação quanto à conservação dos recursos hídricos. Em estudos de Silva et al. (2006), no caso da rega de jardins e limpeza de pátios, no contexto da conservação da água, podem ser adotadas fontes alternativas. Estas alternativas podem ser classificadas como ferramentas da otimização da pegada hídrica azul, minimizando a pressão nos recursos hídricos e favorecendo o estabelecimento de critérios de usos da água.

Em termos de participação percentual no dispêndio mensal o fator consumo humano colaborou com $13,89 \%$ do total de água consumida. Esse aspecto é atribuído às ações humanas realizadas no ambiente de trabalho como utilização de banheiros e higiene pessoal.

Esse é um resultado que vai de encontro com Nascimento e Silva (2015), que em seu estudo, demonstraram que o consumo de água para fins de higiene pessoal encontrou-se entre 65 e $75 \%$ do total de água utilizada. Essa discrepância pode ser justificada pelas peculiaridades do ambiente de trabalho, já que o laboratório comporta 6 funcionários inseridos em $814,65 \mathrm{~m}^{2}$ de área.

Verificou-se que o uso médio real para cada funcionário do laboratório foi de 200 L.dia ${ }^{-1}$. Considerando que esse consumo é variável e ainda sofre influência de instalações e equipamentos hidráulicosanitários, a demanda de água para essas atividades foi calculada em 120 L.dia $^{-1}$. Magalhães et al. (2001) encontraram um consumo médio de 199 L.pessoa ${ }^{-1} \cdot$ dia $^{-1}$, sendo o índice de perdas de 35\% e um consumo efetivo de 129 L.pessoa ${ }^{-1} \cdot$ dia $^{-1}$.

Dentre os tipos de desperdícios de água contabilizados $1,20 \%$ foram caracterizados como de maus hábitos dos usuários, como constatado pela presença de gotejamento em torneiras e vazão excessiva para lavagem de vidraria, bancadas de armazenamento de substrato, piso e janelas. Barreto (2008) verificou em 
estudo na zona oeste de São Paulo que o segundo maior percentual de desperdício de água foi atribuído às perdas de água de pia.

Nunes (2006), em estudos de conservação de água em edifícios comerciais, reportou os maus hábitos entre as principais concepções de causa de elevados volumes de água utilizada e desperdiçada. O diagnóstico preliminar e os valores de consumo de água mostraram ser necessário o emprego de medidas de conservação de água (Tabela 2).

Tabela 2: Relação entre oferta de água e pegada hídrica na produção do fungo M. anisopliae.

\begin{tabular}{lcc}
\hline Atividades & Oferta de água (L.mês ${ }^{-1}$ ) & Demanda hídrica (L.mês ${ }^{-1}$ ) \\
\hline Processo produtivo & 98.220 & 13.200 \\
Atividades de limpeza & 1.500 & 45.000 \\
Consumo humano & 1.440 & 17.280 \\
\hline Total & 101.160 & 37.980 \\
\hline
\end{tabular}

A implantação do reuso de água no sistema de destilação reduziu o consumo de água em $51,21 \%$, o que correspondeu a uma oferta de água de 101.160 L por mês. Grandes volumes de água potável podem ser poupados pelo reuso quando se utiliza água de qualidade inferior (geralmente efluentes pós-tratados) para atendimento das finalidades que podem prescindir desse recurso dentro dos padrões de potabilidade (CETESB, 2015). Nessa perspectiva, o reuso pode ser entendido como "aproveitamento do efluente após uma extensão de seu tratamento, com ou sem investimentos adicionais" (COSTA, 2010). Os estudos de Marisco et al. (2014), evidenciaram o grande potencial de um plano de conservação de água em se tratando dos aparelhos destiladores em diferentes laboratórios.

O processo produtivo colaborou com cerca de $97 \%$ da oferta de água de reuso por mês. Nota-se que o reuso ofertado por essa atividade poderia assumir de maneira individual toda a necessidade mensal de água do laboratório. Esteves (2013) conseguiu com a aplicação de reuso de água aproveitar cerca de 1.000 litros semanais do processo de destilação.

Considerando que o sistema de reuso no processo de destilação é para uso não potável, a água reciclada passou a ser utilizada na limpeza de piso e janelas, lavagem de vidrarias, higienização de bancadas e rega de jardim (Figura 2). Segundo Marisco et al. (2014), o reuso dos efluentes desses equipamentos tem se mostrado viável, uma vez que a qualidade dos mesmos parece permitir o seu uso como água não potável com pouco ou quase nenhum tratamento. Silva (2014) mencionou que devido à alta temperatura $(33,7$ a $58,8^{\circ} \mathrm{C}$ ) sua utilização imediata é dificultada. Dessa maneira, o reservatório aplicado no sistema de reuso do laboratório torna-se um facilitador da perda de calor dentro do sistema implantado.

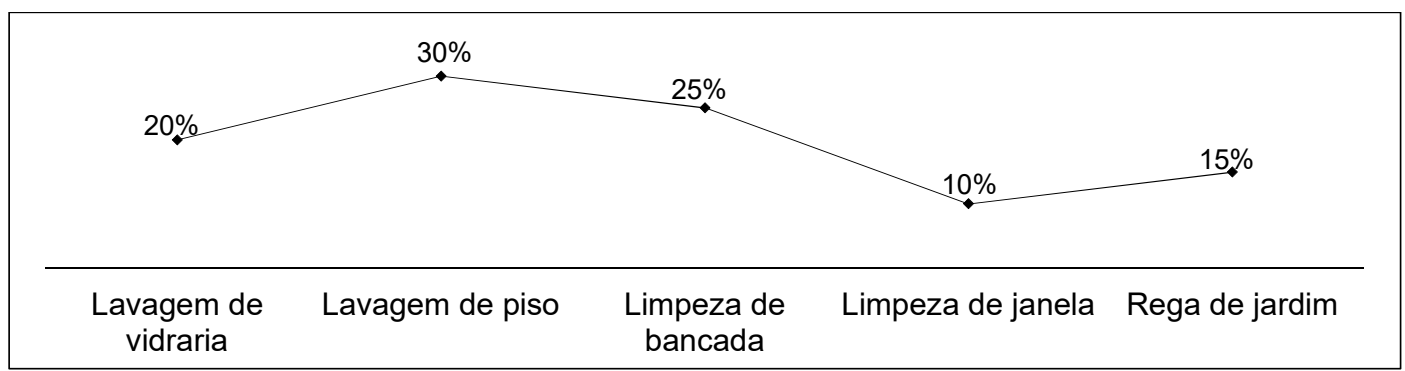

Figura 2: Percentual participativo do reuso nas atividades de limpeza do laboratório de fungos. 
Nota-se que a atividade na qual possui maior demanda de água é a lavagem de piso. Essa é uma prática diária do laboratório e a água reciclada pôde supri-la de maneira integral. Situação semelhante deuse com as atividades de limpeza de bancada e de vidrarias laboratoriais. Em contrapartida, a porcentagem encontrada na prática de limpeza de janelas reflete-se pela sua frequência, que é semanal. A rega de jardim, apesar de não ocorrer diariamente, toma $15 \%$ do total utilizado em virtude da quantidade de água necessária a essa prática. Ressalta-se, nessa vertente, que a utilização de mangueira foi otimizada pela introdução de esguicho, de forma a controlar o fluxo. Estudo de Vimieiro (2005) demonstrou que o emprego desse artifício pode levar a redução de consumo uma vez que o fluxo de água ocorrerá somente quando o usuário estiver utilizando efetivamente a mangueira.

A economia de água no processo de limpeza e consumo humano representaram, conjuntamente, 2,9\%. Essa eficiência poderia ser alavancada se houvesse manutenção e conserto de vazamentos no sistema hidráulico. Ywashima (2005), avaliando vazamento em pavimentos com sanitários, obteve perda de água equivalente a $54,5 \mathrm{~m}^{3} /$ mês e $63,42 \mathrm{~m}^{3} /$ mês. Intervenções e reparos melhorariam a vida útil dos equipamentos hidráulicos, minimizariam as perdas visíveis e invisíveis no sistema hidrossanitário do laboratório e contribuiriam para uma melhor pegada hídrica, importante indicador de sustentabilidade na utilização eficaz da água.

\section{CONCLUSÕES}

Conclui-se que houve desperdício da água utilizada nas atividades de produção do fungo $M$. anisopliae. $\mathrm{O}$ cálculo da pegada hídrica definido como negativo mostrou necessária a implantação de um sistema de reuso de água, a qual diminuiu o consumo e aumentou a oferta hídrica. Pois, a adoção de medidas alternativas de conservação de água frente à disponibilidade e a pegada hídrica atuais é fundamental para a continuidade desse recurso.

A oferta mensal advinda da água reciclada dos destiladores seria capaz de suprir todas as atividades de necessidade de água não potável e potável. Ainda que não opere em circuito fechado, o sistema poderia assumir essa prática depois de testes físico-químicos e microbiológicos que garantissem sua potabilidade. A modificação do sistema para o reuso de água, portanto, apresentou-se como uma técnica eficaz à melhoria da pegada hídrica azul, demonstrando eficiência no uso da água no processo de controle biológico da cultura de cana-de-açúcar.

\section{REFERÊNCIAS ${ }^{1}$}

ABNT. Associação Brasileira de Normas Técnicas. NBR 13969: Tanques sépticos: unidade de tratamento complementar e disposição final dos efluentes líquidos: projeto, discussão e operação. Rio de Janeiro: ABNT, 1997.

ABNT. Associação Brasileira de Normas Técnicas. NBR 5626: Instalação predial de água fria. Rio de Janeiro: ABNT, 1998.
BARRETO, D.. Perfil do consumo residencial e usos finais da água. Ambiente Construído, v.8, n.2, p.23-40, 2008.

BRASIL. Lei $\mathbf{n}^{\circ}$. 9.433, de 08 de janeiro de 1997. Dispõe sobre a Política Nacional de Recursos Hídricos. Brasília: DOU, 1997.

${ }^{1}$ AGRADECIMENTOS: Ao Centro de Desenvolvimento Tecnológico da Usinas Itamarati S/A pela concessão dos dados para a execução deste estudo. 
BERTONCINI, E. I.. Tratamento de efluentes e reuso da água no meio agrícola. Revista Tecnologia e Inovação Agropecuária, v.1, n.1, p.152-169, 2008.

CETESB. Companhia de Tecnologia de Saneamento Ambiental. Padrões de potabilidade da água. CETESB, 2015.

COSTA, R. H. P. G.. Reuso. In: TELLES, D. D'A.; COSTA, R. H. P. G.. Reuso da água: conceitos, teorias e práticas. 2 ed. São Paulo: Blucher, 2010. p.153-207

CREDER, H.. Instalações hidráulicas e sanitárias. 6 ed. Rio de Janeiro: LTC, 2006.

ESTEVES, R. V. R.; CAVALLI, C.; SANTOS, V. L. G.; BUZIN, P. J. W. K.; TUBINO, R. M. C.. Aplicação da metodologia de produção mais limpa no laboratório LEAmet da UFRGS. In: SEMINÁRIO SOBRE TECNOLOGIAS LIMPAS, 5. Anais. Porto Alegre: 2013.

FENG, K.; SIU, Y. L.; GUAN, D.; HUBACEK, K.. Assessing regional virtual water flows and water footprints in the Yellow River Basin, China: A consumption based approach. Applied Geography, v.32, n.1, p.691-701, 2011.

FIESP. Federação das Indústrias de São Paulo. Programa de conservação e reuso de água: PCRA. São Paulo: FIESP, 2011.

MAGALHÃES, C. A. C., MORENO, J. E GALVÃO JÚNIOR, A. C.. Estimativa do consumo per capita em comunidades atendidas pela unidade de negócio do médio Tietê. In: CONGRESSO BRASILEIRO DE ENGENHARIA SANITÁRIA E AMBIENTAL, 21. Anais. João Pessoa: ABES, 2001.

MACHADO, C. J. S.. Reuso de água doce. Revista Eco 21, v.86, n.1, p.1, 2004.

MARISCO, L. V.; FERNANDES, V. C.; CAVAGNI, M. V.; FERNANDES, L. C.; FERNANDES, J. C.. Reuso de efluentes provenientes de aparelhos destiladores. Revista CIATEC, v.6, n.1 p.37-47, 2014.

MARSARO, C. S. G.; GUIMARÃES, P. C.. Avaliação da viabilidade de reutilização da água de refrigeração dos destiladores para lavagem de pipetas. In: SIMPÓSIO DE RECURSOS HÍDRICOS DO NORTE E CENTRO-OESTE, 1. Anais. Cuiabá: 2007.

MATO GROSSO. Secretaria de Estado de Planejamento e Coordenação Geral. Plano de Longo Prazo de Mato Grosso: macro-objetivos, metas globais, eixos estratégicos e linhas estruturantes. In: PRADO, J. G. B.; BERTCHIELI, R.; OLIVEIRA, L. G.. Plano de Longo Prazo de Mato Grosso. Cuiabá: Central de Texto, 2012.

MIERZWA, J. C.; HESPANHOL, I.. Água na Indústria: Uso Racional e Reuso. São Paulo: Oficina de Textos, 2005.

MORAES D. S. L.; JORDÃO B. Q.. Degradação de recursos hídricos e seus efeitos sobre a saúde humana. Revista Saúde Pública, v.36, n.1, p.370-374, 2002.

NASCIMENTO, S. S.; SILVA, M. C.. Educação ambiental na UFMG - 1998-2011: pesquisas acadêmicas. Revista da Avaliação do Ensino Superior, v.20, n.2, p.401-422, 2015.
NUNES, R. T. S.. Conservação da Água em Edifícios

Comerciais: Potencial de uso racional e reuso em shopping center. Dissertação (Mestrado em Engenharia) -

Universidade Federal do Rio de Janeiro, Rio de Janeiro, 2006.

OLIVEIRA, L. H.. Metodologia para a implantação de programa de uso racional da Água em edifícios. Tese (Doutorado em Engenharia de Construção Civil) Universidade de São Paulo, São Paulo, 1999.

ROMAGUERA, M.; HOEKSTRA, A. Y.; SU, Z.; KROL, M. S.; SALAMA, M. S.. Potencial of using remote sensing techniques for global assessment of water footprint of crops. Journal Remote Sensing, v.2, n.1, p.1177-1196, 2010.

SILVA, G. S.. Programas permanentes de uso racional da água em campi universitários: o programa de uso racional da água da Universidade de São Paulo. Dissertação (Mestrado em Engenharia Agrícola) - Universidade de São Paulo, 2004.

SILVA, G. S.; TAMAKI, H. O.; GONÇALVES, O. M. Implementação de programas de uso racional da água em campi universitários. Ambiente Construído, v.6, n.1, p. 4961, 2006.

SILVA, V. P. R.; ALEIXO, D. O.; DANTAS NETO, J.; MARACAJÁ, K. F. B.; ARAÚJO, L. E.. Uma medida de sustentabilidade ambiental: Pegada hídrica. Revista Brasileira de Engenharia Agrícola e Ambiental, v.17, n.1, p.100-105, 2013.

TOMAZ, P.. Previsão do consumo de água: interface das instalações prediais de água e esgoto com os serviços públicos. São Paulo: Navegar, 2000.

TOMAZ, P. Economia de água para empresas e residências: um estudo atualizado sobre o uso racional da água. São Paulo: Navegar, 2001.

TSUTIYA, M. T.. Abastecimento de água. São Paulo: Departamento de Engenharia Hidráulica e Sanitária da Escola Politécnica da Universidade de São Paulo, 2005.

VIMIEIRO, G. V.. Educação ambiental e emprego de equipamentos economizadores na redução do consumo de água em residências de famílias de baixa renda e em uma escola de ensino fundamental. Tese (Doutorado em Saneamento, Meio Ambiente e Recursos Hídricos) Universidade Federal de Minas Gerais, Belo Horizonte, 2005.

WREGE, M.. A ética da água. InformANDES, v.96, n.1, 2000.

YWASHIMA, L. A.. Avaliação do uso de água em edifícios escolares públicos e análise de viabilidade econômica da instalação de tecnologias economizadoras nos pontos de consumo. Dissertação (Mestrado em Engenharia Civil) Universidade Estadual de Campinas, Campinas, 2005.

YU, Y.; HUBACEK, K.; FENG, K. GUAN, D.. Assessing regional and global water footprints for the UK. Ecological Economics, v.69, n.2, p.1140-1147, 2010.

ZHAO, X.; CHEN, B.; YANG, Z. F.. National water footprint in an input-output framework-A case study of China 2002. Ecological Modeling, v.220, n.1, p.245-253, 2009. 